\title{
The Clinicopathologic Evaluation of Eosinophilic Cholecystitis: A Retrospective Observational Study in a Tertiary Care Center
}

\author{
Senay Erdogan-Durmus ${ }^{1 *}$, Hilal Balta ${ }^{2}$, Sevilay Ozmen ${ }^{3}$, Ilknur Calik ${ }^{2}$, Yusuf Can and Ali Kurt ${ }^{4}$ \\ 'Department of Pathology, Istanbul Basaksehir Cam and Sakura City Hospital, İstanbul, Turkey \\ ${ }^{2}$ Department of Pathology, Firat University Medical Facult, Elazığ, Turkey \\ ${ }^{3}$ Department of Pathology, Atatürk University Medical Faculty, Erzurum, Turkey \\ ${ }^{4}$ Department of Pathology, Erzurum Region Training and Research Hospital, Erzurum
}

\begin{abstract}
Background: Eosinophilic cholecystitis (EC) is a rare form of cholecystitis that is diagnosed histopathologically. The aim of this study to evaluate the patient demographic features, laboratory findings and histopathologic characteristics of EC. The aim of this study to evaluate the patient demographic features, laboratory findings and histopathologic characteristics of EC.

Design and Setting: Retrospective observational study conducted in Erzurum, Turkey.

Methods: Between June 2014 and June 2017 3,178 cholecystectomy specimens were reviewed retrospectively. Nineteen EC cases were included to the study. Parameters such as sex, age, clinical information, laboratory findings were obtained from information system of hospital. And the data of EC group were compared with a control group of 50 non-specific chronic cholecystitis patients (CC).

Result: Out of 3,178 cholecystectomy specimens, 19 cases were diagnosed as EC (0.59\%). Patients' age ranged from $22-84$ years and female to male ratio was 1.7:1. The average eosinophil count was $139.7 \mathrm{U} / \mathrm{L}$ in EC group. 11.7\% of EC group had high level for eosinophils. In the EC group there were statistical significances between high eosinophil percentages and aspartate transaminase (AST), alkaline phosphatase $(\mathrm{ALP})$ values $(\mathrm{P}<0.05, \mathrm{P}<0.05)$. The EC group eosinophil levels were slightly higher than CC. Histopathologically, dense, severe infiltrates composed of eosinophils were seen.

Conclusion: EC is a rare entity that can only diagnosed by histopathology. There were certain high levels in liver function tests and eosinophils in correlation with non-specific cholecystitis. Although the presentation may be similar to non-specific cholecystitis, if a postoperative histopathological diagnosis of EC is made, then the patient must be investigated thoroughly to rule out other associated disease conditions.
\end{abstract}

Keywords: Cholecystitis, Eosinophilia, Cholelithiasis, Histopathology

\section{Introduction}

Eosinophilic cholecystitis (EC) is a rare form of cholecystitis that was first described in $1949 .{ }^{[1]}$ Although cases reported that associated with parasitosis, hyper-eosinophilic syndrome, infections, drugs and some medicinal herbs the etiology is often unknown. Clinical symptoms and laboratory findings do not distinguish from those of other causes of cholecystitis, except peripheral eosinophilia which is sometimes observed. Diagnosis is histological and performed after analysis of the surgical specimen. ${ }^{[1,2]}$

It is characterized histologically by a marked infiltrate composed predominantly or almost exclusively of eosinophils. It is diagnosed when cellular infiltrate consists of more than $90 \%$ eosinophils. ${ }^{[2]}$ The eosinophils can infiltrate the mucosa, muscular layer, serosa or all of them. ${ }^{[3]}$

There are only few case reports about EC in literature. Large case series or studies which evaluate the clinical, histological features and laboratory findings of EC are also infrequent.

The aim of this study is to evaluate the demographic features, laboratory findings such as peripheral eosinophilia, medical history such as chronic disease, drugs and histopathologic features of EC.

\section{Materials and Methods \\ Patients}

Between June 2014 and June 2017, the histopathologic features of 3,178 cholecystectomy specimens were reviewed retrospectively. Nineteen of $3,178(0.59 \%)$ cases which were diagnosed as EC and included in the study. Fifty non-specific cholecystitis cases (CC) cases were selected randomly for the control group. Parameters such as sex, age of the patients, clinical information and laboratory findings were obtained from information system 
of hospital. The data of the EC group were compared with the $\mathrm{CC}$ group.

\section{Statistical Analysis}

Data analysis performed using the SPSS 20.0 program. Descriptive statistics for the evaluation of results were shown in the form of mean \pm standard deviation, the nominal variables were shown as the number of cases and (\%). We used Pearson Chi-Square, Fisher Exact validation tests and Spearman and Pearson's correlation test for numerical data. Significance level was defined as a $\mathrm{P}<0.05$.

\section{Ethic}

The study was found appropriate ethically according to the ethical committee of Erzurum Region Training and Research Hospital with the decision number 2017/07-43 and date 7th June 2017.

\section{Result}

The the mean age of EC group was $44.63 \pm 16.59$ (age range 22-84). Seven patients were under 40 whereas 12 patients over 40 . There was a female predominance. Twelve out of 19 patients (63.1\%) were females and 7 of them $(36.9 \%)$ were males. In CC, the mean age was 49.72 \pm 13.76 . There was a female predominance, with a female to male ratio of $3,1: 1$.

All cases of EC group applied with abdominal pain. The mean level for aspartate transaminase (AST) was $54.5 \mathrm{U} / \mathrm{L}$ (range 5-34 U/L), for alanine transaminase (ALT) was 69.1 $\mathrm{U} / \mathrm{L}$ (range $0-55 \mathrm{U} / \mathrm{L}$ ) and for alkaline phosphatase (ALP) was $125.1 \mathrm{U} / \mathrm{L}$ (range $40-150 \mathrm{U} / \mathrm{L}$ ). The number of cases and percentages that have high levels of AST, ALT and ALP in EC and CC were shown in Table 1. In CC group $80 \%$ of the cases were admitted with abdominal pain. The mean level for AST, ALT and ALP were 25.3 U/L, 33.4 $\mathrm{U} / \mathrm{L}, 96.8 \mathrm{U} / \mathrm{L}$, respectively.

The mean level for white blood cells (WBC) in hemogram was $9,034 \mathrm{U} / \mathrm{L}$ (range 3,700-10,100 U/L). High percentages of $\mathrm{WBC}$ in $\mathrm{EC}$ and $\mathrm{CC}$ were shown in Table 2. The average eosinophil count was $139.7 \mathrm{U} / \mathrm{L}$ (range: $0-400 \mathrm{U} / \mathrm{L}$ ) in the
EC group and $128.4 \mathrm{U} / \mathrm{L}$ in CC. In the EC group, 11.7\% of patients had high level for eosinophils, while $4 \%$ of patients in the CC group had it.

In the EC group there were statistical significances between high eosinophil percentages and AST, ALP values $(\mathrm{p}<$ $0.05, \mathrm{p}<0.05)$ while there was no significance between eosinophil percentages and ALT values ( $p>0.05)$. There was no statistically significant difference between the two groups for ALT, AST, ALP and WBC values. In the EC group eosinophil levels were slightly higher than CC group, but there was no significance statistically ( $p>0.05$ ).

In the EC group, 15 out of 19 cases ( $78.9 \%$ ) had gallstones in ultrasonographic and gross examination while one of them had biliary mud. On gross examination, the gall bladders' lengths were 5.5-19 centimeters and wall thicknesses were 2-15 mm (mean $5.2 \mathrm{~mm}$ ). In the EC group there was statistically significant correlation between wall thickness and WBC, ALT levels ( $p<0.05, \mathrm{p}<0.05$, respectively). In the CC group, 30 out of 50 cases $(60 \%)$ had gallstones. On gross examination, the gall bladders' lengths were 4-11 centimeters and wall thicknesses were 1-10 millimeters (mean 3.5 millimeters). Although the wall thickness in the EC group was relatively high, no statistical difference was found.

Histopathologically, in the EC group dense infiltrates composed of more than $90 \%$ eosinophils were seen. In the majority of cases infiltrates were transmural (Fig. 1a-d). In the CC group, chronic inflammation was observed accompanied by lymphocytes, plasma cells and very infrequent eosinophils, histopathologically.

Retrospective analysis of patients' medical histories did not indicate any known etiology. One of patients in the EC group had used paliperidone for paranoid schizophrenia and one of them had have used salbutamol for chronic obstructive pulmonary disease.

From the hospital system follow-up of the patients between 6-36 months (mean 12.7 months) was reached. No other eosinophilic inflammation was detected.

Table 1: Changes of laboratory findings in eosinophilic cholecystitis (EC) and non- specific chronic cholecystitis (CC) control groups.

\begin{tabular}{|l|l|l|}
\hline & EC $(\mathbf{n} / \%)$ & CC $(\mathbf{n} / \%)$ \\
\hline High AST levels & $7 / 36.8 \%$ & $7 / 14 \%$ \\
\hline High ALT levels & $8 / 42.1 \%$ & $6 / 12 \%$ \\
\hline High ALP levels & $5 / 36.3 \%$ & $7 / 14 \%$ \\
\hline
\end{tabular}

$A S T=$ aspartate transaminase $; A L T=$ alanine transaminase $; A L P=$ alkaline phosphatase. 
Table 2: High percentages of white blood cells (WBC) in eosinophilic cholecystitis (EC) and non-specific chronic cholecystitis (CC) control groups.

\begin{tabular}{|l|l|l|}
\hline & EC $(\mathbf{n} / \%)$ & CC $(\mathbf{n} / \%)$ \\
\hline High percentages of neutrophils & $6 / 31.5 \%$ & $10 / 20 \%$ \\
\hline High percentages of monocytes & $4 / 21.0 \%$ & $7 / 14 \%$ \\
\hline High percentages of eosinophills & $2 / 11.7 \%$ & $2 / 4 \%$ \\
\hline Total & $\mathbf{1 2 / 7 0 . 5} \%$ & $\mathbf{1 9 / 3 8} \%$ \\
\hline
\end{tabular}

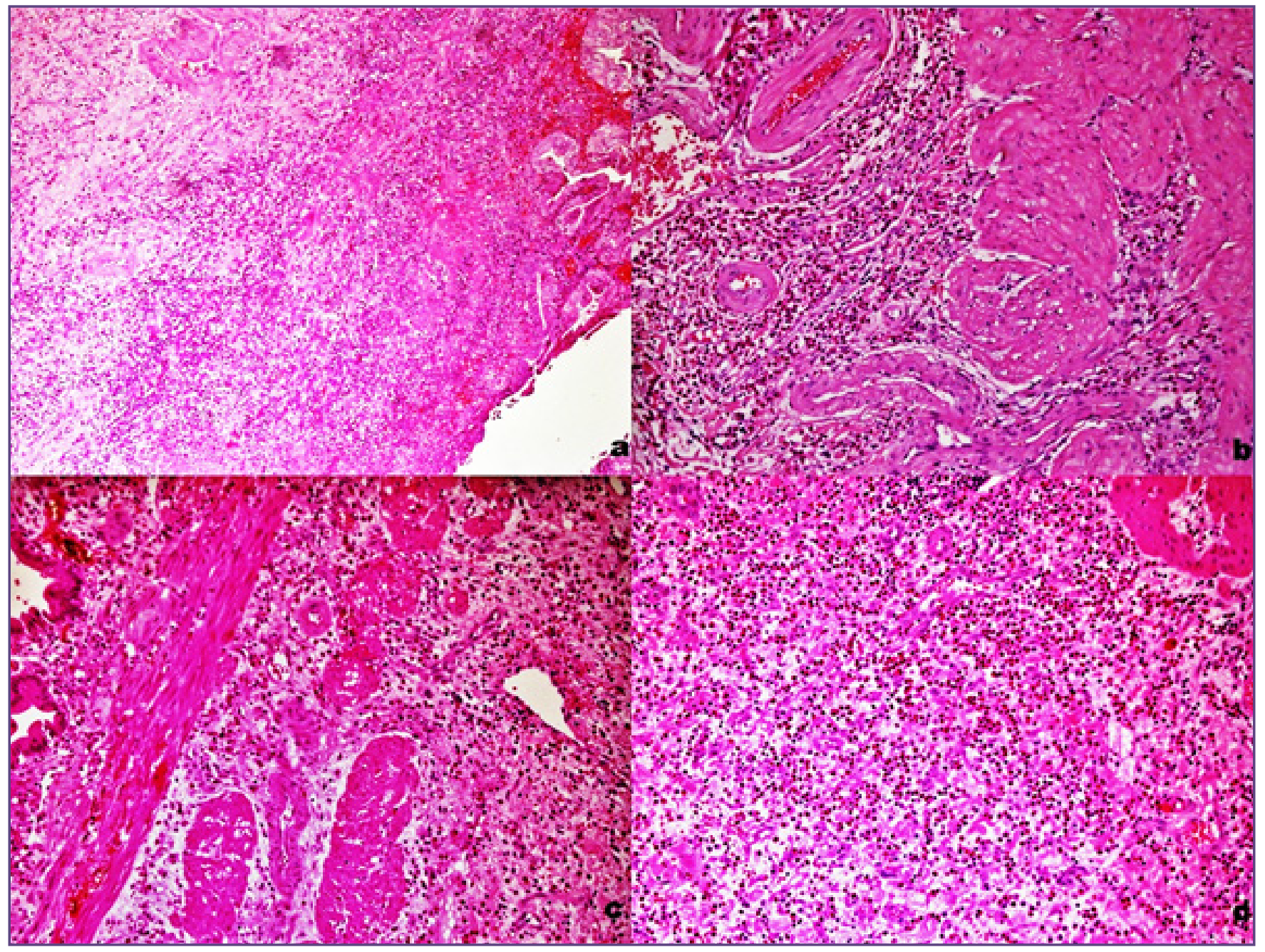

Fig 1a-d: Severe eosinophilic inflammation in mucosal layer, among muscle fibers and transmural inflammation was seen in different cases (a- H\&Ex100, b- H\&Ex200, c- H\&Ex200, d- H\&Ex200).

\section{Discussion}

Eosinophilic cholecystitis is an infrequent form of cholecystitis which incidence ranges from $0.25 \%$ to $6.4 \%$. ${ }^{[3,4]}$ In the present study, EC was diagnosed in 19 of 3,178 cholecystectomy specimens $(0.59 \%)$, which is similar to the results of other reported series. ${ }^{[3,4]}$

The etiology of EC is not clear. It may be associated with peripheral eosinophilia syndromes, including allergic conditions such as asthma, atopic diseases, hypereosinophilic syndrome, parasitic diseases of the liver and biliary tract and conditions such as gallstones, acalculous cholecystitis. ${ }^{[4-6]}$ Also, hypersensitivity to antibiotics, drugs and herbal medicine was found responsible in the etiology and has been reported before. ${ }^{[4]}$ The most accepted theory is an allergic reaction against an antigen in the bile. Antigen stimulate mast cell degranulation, and this induce releasing eosinophilic chemotactic factors, leukotrienes, 
and other platelet-activating factors. This mechanism can explain excessive eosinophilic infiltration in the wall of gall bladder. ${ }^{[7-9]}$ Despite that, the etiology of most cases in the literature is unknown. ${ }^{[3,4,10,11]}$

In our study, retrospective analysis of our cases histories and investigation did not reveal any known etiology. One of our patients had have used for paranoid schizophrenia and one of them had have used salbutamol for chronic obstructive pulmonary disease. Although there are studies showing that salbutamol increases eosinophilia in sputum, paliperidone and salbutamol have no relationship with EC defined yet. ${ }^{[12,13]} \mathrm{A}$ valuable factor was not found in this study for EC etiology.

Eosinophilic cholecystitis can't be separated clinically from other forms of cholecystitis. All cases present with abdominal pain, especially localized in right upper quadrant as in our patients. ${ }^{[4,11]}$ Therefore, the diagnosis only can made by histological analysis. The diagnosis of EC should be restricted to massive infiltrations of eosinophils (more than $90 \%$ eosinophils) in microscopic examination. When the infiltrate be formed of $50-75 \%$ eosinophils along with other inflammatory cells it is diagnosed as lympho-eosinophilic cholecystitis. ${ }^{[2,3,4]}$ Similarly, in our study mostly transmural, dense, diffuse eosinophilic infiltrates and few lympocytes were seen in EC cases, histopathologically.

In some studies researchers found that patients with acalculous cholecystitis contain a higher percentage of eosinophils, histopathologicallly than in patients with gallstones. ${ }^{[2-4,7,11]}$ In contrast, $78.9 \%$ of our cases had biliary stones and one of them had biliary mud.

Peripheral blood eosinophilia in EC patients has been reported in some studies. ${ }^{[6,14,15]}$ In a previous study researchers found high levels for eosinophils in 4 of 15 EC patients. ${ }^{[16]}$ In other studies peripheral eosinophilia was noted in $20 \%$ and $9 \%$ of EC cases. ${ }^{[6,14]}$ In the present study 2 patients (11.7\%) had high level for eosinophils in the EC group. Differently from literature, in our study we examined the WBC levels and percentages of neutrophils, monocytes and eosinophils. And we found high levels for WBC in five EC cases. High percentages of neutrophils, monocytes and eosinophils were found in $31.5 \%, 21 \%$ and $11.7 \%$ of EC cases, respectively. And high percentages of neutrophils, monocytes and eosinophils were found in $20 \%, 14 \%$ and $4 \%$ of CC group.

Differently from literature, our study searched liver function tests such as AST, ALT and ALP. And there were statistical significances between high eosinophil percentages and AST, ALP values $(\mathrm{p}<0.05, \mathrm{p}<0,05)$.
Eosinophilic infiltration may be associated with multiple organ involvement. Eosinophilic infiltration of the other parts of gastrointestinal tract may occur in eosinophilic cholangiopathy, eosinophilic gastroenteritis, eosinophilic granulomatous hepatitis. Especially in hypereosinophilic syndrome eosinophilic ascites may occur, too. ${ }^{[16,17]} \mathrm{EC}$ can also be seen with eosinophilic cystitis. ${ }^{[18]}$

\section{Limitations}

Although, in our study the incidence of EC similar to literature $(0.59 \%)$, scarcity of the case number $(\mathrm{N}: 19)$ limited the study. For instance; some statistical calculations could not be made. May be more patients can obtain if the studies spread over much longer period of time.

\section{Conclusion}

EC is a relatively uncommon entity in which can only diagnosed by histopathology. It is more common in females. Peripheral eosinophilia is not uncommon. There was certain increase in liver function tests. Although the presentation may be similar to general cholecystitis, if a postoperative histopathological diagnosis of EC is made, then the patient must be investigated thoroughly to rule out other associated disease conditions. That can change patients' managements. Further researches seem to be necessary with larger patient groups.

\section{Acknowledgements}

No.

\section{Funding}

No.

\section{Competing Interests}

No.

\section{References}

1. del-Moral-Martínez M, Barrientos-Delgado A, CrespoLora V, Cervilla-Sáez-de-Tejada ME, Salmerón-Escobar J. Eosinophilic cholecystitis: an infrequent cause of acute cholecystitis. Rev Esp Enferm Dig. 2015;107:45-7. P

2. Choudhury M, Pujani M, Katiyar Y, Jyotsna PL, Rautela A. Idiopathic eosinophilic cholecystitis with cholelithiasis: a report of two cases. Turk Patoloji Derg. 2014;30:142-4.

3. Jesserun J, Pambuccian S. Infectious and inflammatory disorders of the gallblader and extrahepatic biliary tract. In: Odze RD, Goldblum JR, editors. Surgical Pathology of the GI Tract, Liver, Biliary Tract and Pancreas. 3rd ed. China: Elsevier Saunders; 2015. 995-1021.

4. Yeom SS, Kim HH, Kim JC, et al. Peripheral eosinophilia - is it a predictable factor associated with eosinophilic cholecystitis?. Korean J Hepatobiliary Pancreat Surg 2012;16:65-9. 
5. Russell CO, Dowling JP, Marshall RD. Acute eosinophilic cholecystitis in association with hepatic echinococcosis. Gastroenterology. 1979;77:758-60.

6. Hepburn A, Coady A, Livingstone J, Pandit N. Eosinophilic cholecystitis as a possible late manifestation of the eosinophilia-myalgia syndrome. Clin Rheumatol. 2000;19:470-2.

7. Adusumilli PS, Lee B, Parekh K, Farrelly PA. Acalculous eosinophilic cholecystitis from herbal medicine: a review of adverse effects of herbal medicine in surgical patients. Surgery. 2002;131:352-6.

8. Cerdán Santacruz C, Martín Antona E, Martín GarcíaAlmenta E, Díez Valladares L, Torres García AJ. Eosinophilic cholecystitis after elective cholecystectomy for gallbladder stones. Cir Esp. 2013;91:460-1.

9. Saks K, Amjadi D, D’Souza SL. Eosinophilic cholecystitis and enteritis associated with ampullary stenosis. Clin Gastroenterol Hepatol. 2017;15:A23-A24.

10. Shakov R, Simoni G, Villacin A, Baddoura W. Eosinophilic cholecystitis, with a review of the literature. Ann Clin Lab Sci. 2007;37:182-5.

11. Singh DK, Shankar R, Gondal R, Malhotra V, Mishra P. Idiopathic eosinophilic cholecystitis with cholelithiasis: A case report and review of literature. Internet Journal of Surgery. 2008;16:2.
12. Jarema M, Bieńkowski $\mathrm{P}$, Heitzman J, Parnowski $\mathrm{T}$, Rybakowski J. Paliperidone palmitate: effectiveness, safety, and the use for treatment of schizophrenia. Psychiatr Pol 2017;26;51(1):7-21.

13. Gordon JR, Swystun VA, Li F, Zhang X, Davis BE, Hull P, Cockcroft DW. Regular salbutamol use increases CXCL8 responses in asthma: relationship to the eosinophil response. Eur Respir J. 2003;22(1):118-26.

14. Tajima K, Katagiri T. Deposits of eosinophil granule proteins in eosinophilic cholecystitis and eosinophilic colitis associated with hypereosinophilic syndrome. Dig Dis Sci. 1996;41:282-8.

15. Kim YH. Eosinophilic cholecystitis in association with clonorchis sinensis infestation in the common bile duct. Clin Radiol. 1999;54:552-4.

16. Khan S, Hassan MJ, Jairajpuri ZS, Jetley S, Husain M. Clinicopathological Study of Eosinophilic Cholecystitis: Five Year Single Institution Experience. J Clin Diagn Res. 2017;11:EC20-EC23.

17. Kumar V, Verma R, Dawan S, Choudhary M, Verma S, Dhaka JP. Incidence of Eosinophilic Cholecystitis in Northwestern Region of Rajasthan: A Study in Consecutive 867 Cholecystectomies. Int J Sci Stud. 2016;4:150-3.

18. Mallat F, Hmida W, Mestiri S, et al. Eosinophilic Cystitis with Eosinophilic Cholecystitis: A Rare Association. Case Rep Urol. 2013;2013:146020.

*Corresponding author:

Senay Erdogan-Durmus, Erzurum Region Training and Research Hospital

Phone: +91 905327371881

Email: senayerdgn@gmail.com

Date of Submission : $03 / 03 / 2021$

Date of Final Revision : 20/04/2021

Date of Acceptance : 20/05/2021

Financial or other Competing Interests: None.

Date of Publication : 30/06/2021 